\title{
Additive effects of emotional, endogenous, and exogenous attention: Behavioral and electrophysiological evidence
}

Tobias Brosch ${ }^{1,2,3}$, Gilles Pourtois ${ }^{4}$, David Sander ${ }^{2,3}$, and Patrik Vuilleumier ${ }^{2,5}$

\author{
${ }^{1}$ Department of Psychology, New York University, New York, USA \\ ${ }^{2}$ Swiss Center for Affective Sciences, University of Geneva, Switzerland \\ ${ }^{3}$ Department of Psychology, University of Geneva, Switzerland \\ ${ }^{4}$ Department of Experimental Clinical and Health Psychology, Ghent University, \\ Belgium \\ ${ }^{5}$ Department of Neuroscience, University Medical Center, Geneva, Switzerland
}

Corresponding author:

Tobias Brosch

Department of Psychology

New York University

6 Washington Place

New York, NY 10003

tobias.brosch@nyu.edu

+1 (212) 998-3720 


\begin{abstract}
Selective attention is not a unitary construct, but is composed of several processes. Attention selection may be guided by low-level stimulus properties, by the emotional value of the stimulus, or more voluntarily by the goals and plans of the observer. Whether these three systems operate independently during attention selection or not remains a debated question. We report results from two studies investigating the extent to which these different attention mechanisms may interact with one another. Using a standard dot probe paradigm wherein effects of exogenous, emotional, and endogenous attention were orthogonally manipulated, we found attentional facilitation effects for each component, indicated by faster decision times for validly, as opposed to invalidly cued targets. Moreover, results confirmed that these three attentional effects added up in a linear fashion. Complementing ERP results allowed us to disentangle the respective contributions of the two reflexive, bottom-up attention processes (exogenous vs. emotional) by showing non-overlapping temporal loci for attentional effects related either to low-level physical properties or the emotional content of the stimulus. These findings suggest that multiple separate attention mechanisms can operate simultaneously to yield a rapid and efficient visual processing of various classes of potentially relevant stimuli.
\end{abstract}

Key words: Attention; Emotion; Exogenous; Endogenous; Dot Probe; EEG 


\section{Additive effects of emotional, endogenous, and exogenous attention: Behavioral and electrophysiological evidence}

\section{Introduction}

Due to the capacity limits of the human brain (Duncan, 1980; Marois \& Ivanoff, 2005), not all incoming environmental stimulation can be processed in parallel and evaluated thoroughly. To allow for a rapid and efficient analysis of behaviorally important information in the environment, dedicated attention systems therefore serve to select a subset of all possible stimuli for more in-depth processing and preferential access to conscious awareness (Driver, 2001). Attentional selection is guided by stimulus-related as well as by observer-dependent effects. Therefore, attention selection is not a unitary construct, but distinct functional subprocesses related to different selection criteria have been put forward, and their respective properties and contributions to attentional selection mechanisms have been isolated using both behavioral and brain-imaging methods.

Exogenous attention refers to effects driven by the intrinsic low-level salience of sensory inputs (Egeth \& Yantis, 1997; Theeuwes, 1991; Wolfe \& Horowitz, 2004). Low-level physical properties (such as stimulus intensity, color, or size) may trigger an involuntary, stimulus-driven, bottom-up attention process. By contrast, endogenous attention refers to a voluntary top-down process, initiated by internal states and conscious expectations for a specific object or location (Desimone \& Duncan, 1995; Posner, Snyder, \& Davidson, 1980). This process selects stimuli important to the current behavior and goals of the organism. According to a recent neuro-cognitive model of attention, both endogenous and exogenous attention primarily implicate fronto-parietal networks of cortical regions (Corbetta, Patel, \& Shulman, 2008; Corbetta \& Shulman, 2002; see also Peelen, Heslenfeld, \& Theeuwes, 2004), with endogenous attention control being exerted by interactions of dorsal regions such as the intraparietal sulcus (IPS) and the frontal eye fields (FEF), and exogenous reorienting of the attentional focus mediated by more ventral regions in the right hemisphere such as the right ventral frontal cortex (VFC) and temporo-parietal junction (TPJ).

In addition to endogenous and exogenous attention mechanisms, a large body of neuroimaging and behavioral research suggests that the emotional relevance of a stimulus can also constitute an important feature influencing selection by attention (Compton, 2003; Lang, Bradley, \& Cuthbert, 1997; Vuilleumier, 2005; Williams \& Gordon, 2007). Unlike endogenous processes, this mechanism operates without conscious control, but unlike exogenous processes, it is often modulated by internal affective states such as state or trait anxiety (e.g., Bishop, Duncan, Brett, \& Lawrence, 2004; Bishop, Jenkins, \& Lawrence, 2007; Fox, Russo, Bowles, \& Dutton, 2001). Various emotional stimuli such as angry facial expressions, snakes, babies or emotionally arousing words have been found to be processed preferentially and to modulate attention brain mechanisms (see, e.g., Brosch, Sander, Pourtois, \& Scherer, 2008; Öhman, Flykt, \& Esteves, 2001). It has been suggested that dedicated neural circuits might subserve this form of emotional attention (Vuilleumier, 2005; Vuilleumier \& Brosch, 2009). In this model, the amygdala, a limbic region critically involved in the processing of emotional information (LeDoux, 2000) is thought to play a critical role by modulating the processing of incoming sensory stimuli through 
direct feedback projections to visual cortex (Amaral, Behniea, \& Kelly, 2003) and biasing signals to fronto-parietal attention regions (Pourtois, Thut, Grave de Peralta, Michel, \& Vuilleumier, 2005).

The interplay of emotional attention with other attentional mechanisms has recently generated a bulk of studies and debates in affective neuroscience (Pessoa, 2005; Vuilleumier, 2005; Vuilleumier \& Driver, 2007). Several studies have demonstrated parallel and additive influences of emotional stimulus content and endogenous attention allocation on the brain responses toward a stimulus. For example, by manipulating both factors orthogonally in fMRI studies, it was found that the neural response to faces in face-sensitive regions of the visual cortex (Vuilleumier, Armony, Driver, \& Dolan, 2001) and to voices in voice-sensitive areas of the auditory cortex (Grandjean et al., 2005) was modulated by each factor independently, and that amygdala activation by stimulus emotionality was unaffected by endogenous attention (Sander et al., 2005; Vuilleumier et al., 2001), consistent with the presumed role of the amygdala in driving emotional enhancement (see also Vuilleumier, Richardson, Armony, Driver, \& Dolan, 2004). Likewise, additive effects of emotional and endogenous attention have been demonstrated with event-related potentials (ERPs) to complex emotional scenes presented at attended or unattended locations in visual hemifields (Keil, Moratti, Sabatinelli, Bradley, \& Lang, 2005; see also Pourtois, Spinelli, Seeck, \& Vuilleumier, 2010). Other studies have reported conflicting results, finding that the influence of emotional distractors on attentional competition depends on the availability of endogenous attention capacities (e.g., Pessoa, McKenna, Gutierrez, \& Ungerleider, 2002; Silvert et al., 2007), and that directing attention away from emotional stimuli may reduce amygdala responses in tasks in which attentional load is particularly high (Pessoa, Padmala, \& Morland, 2005; Silvert et al., 2007), thus possibly also reducing the impact of emotion on perceptual processing.

So far, mainly the interplay of emotional and endogenous attention has been investigated (e.g., Pessoa et al., 2002; Vuilleumier et al., 2001), whereas the relation between emotional and exogenous attention has generally been overlooked.

Conceptually, emotional attention and exogenous attention may appear more similar to each other than they are to endogenous attention, as both are presumably reflexive processes operating independently of current voluntary goals. Accordingly, one might hypothesize that a common attention system might drive the reflexive prioritization of both physically salient and emotionally salient stimuli. On the other hand, emotional attention has been suggested to rely on specialized neural circuitry involving the amygdala (Vuilleumier, 2005), and is modulated by internal affective states (Bishop, 2007; Fox et al., 2001). Based on this evidence, one might predict that emotional and exogenous attention reflect different sources of modulations on sensory processing that operate independently of one another. However, there is not much empirical research addressing this issue. To our knowledge, only one previous behavioral study tackled a related question (Tipples \& Sharma, 2000), showing that exogenous attention orienting was not affected by the emotional value of centrally presented pictures.

In the current set of studies, we investigated the interplay of emotional, exogenous, and endogenous attention at the behavioral and neural level. We used variants of the dot probe task (MacLeod, Mathews, \& Tata, 1986), a standard task for the measurement of emotional attention. In this task (see Figure 1), participants usually 
respond to the presentation of a unilateral target, which is preceded by a pair of stimuli, one of them being emotional, the other being neutral. If the target appears at the location previously occupied by the emotional stimulus (valid trials), participants typically show better perception and faster detection of the target stimuli (Brosch, Sander, \& Scherer, 2007; Phelps, Ling, \& Carrasco, 2006; Pourtois, Grandjean, Sander, \& Vuilleumier, 2004). Such findings are consistent with a reflexive orienting induced by emotional attention, although they may also be explained in terms of a more prolonged disengagement from the emotional stimulus in invalid trials (Fox, Russo, \& Dutton, 2002). In our study, we modified this standard task by including additional manipulations of exogenous (Experiments 1 and 2) and endogenous (Experiment 1) attention.

\section{Experiment 1}

In Experiment 1, we combined an emotional attention manipulation with an orthogonal manipulation of exogenous spatial attention, triggered by a sudden and non-predictive bright flash (Berger, Henik, \& Rafal, 2005), and a concurrent orthogonal manipulation of endogenous attention, triggered by an arrow presented at fixation (Posner et al., 1980, see Figure 1). In exogenous valid trials, the target appeared at the location of the bright flash. In endogenous valid trials, the arrow pointed to the location where the target appeared in most of the trials $(70 \%$ in our paradigm). Endogenous spatial orienting was thus based on the symbolic meaning of the arrow and its predictive value (Jonides, 1981; Theeuwes, 1991). Importantly, previous work has suggested that the different attention components have different time courses. Shifts of exogenous attention have typically been reported with a stimulus onset asynchrony (SOA) not exceeding 50-100 ms, whereas shifts of endogenous attention have a longer onset and yield maximal facilitation effects at SOAs between 400 and $800 \mathrm{~ms}$ (Shepherd \& Muller, 1989). Shifts of emotional attention have been observed with SOAs ranging from 14 to 500 ms (Bradley, Mogg, \& Millar, 2000; Mogg \& Bradley, 1999). Thus we used two different SOAs (a short one $-100 \mathrm{~ms}$, and a much longer one $-800 \mathrm{~ms}$ ) to temporally disentangle these different subcomponents. Furthermore, we introduced the endogenous cueing manipulation earlier during the trial sequence than the two other manipulations, to provide sufficient time for the unfolding of the voluntary attention shift even with the short SOA (see Figure 1 and Method section). We surmised that effects of emotional and exogenous attention should predominantly occur with the short SOA, whereas effects of endogenous attention should be observed at the short and long SOAs.

\subsection{Methods}

\subsubsection{Participants}

Thirty-four University of Geneva students ( 28 females, mean age 22.7 years) participated in the experiment for course credit. All participants were right-handed, had normal or corrected-to-normal vision, and no history of psychiatric or neurological diseases.

\subsubsection{Procedure and Data Analysis}

The experiment consisted of one practice block of 10 trials, followed by four experimental blocks of 220 trials each. A fixation cross was presented continuously at the center of the screen. During each trial, two thin frames were shown on the screen 
continuously, one in the left and one in the right visual field (LVF and RVF, respectively). The frames were $7 \times 10 \mathrm{~cm}$ on the screen and presented with $15 \mathrm{~cm}$ between fixation cross and image center. Participants were seated in front of the screen at a viewing distance of $100 \mathrm{~cm}$, resulting in a visual angle of $8.5^{\circ}$ between the fixation cross and the center of the frame. Each trial started with the fixation cross only presented for a random interval between 750 and $1250 \mathrm{~ms}$. Then the endogenous attention cue, consisting of a centrally presented arrow (at the position of the fixation cross) pointing to the left or the right, was presented for $200 \mathrm{~ms}$, followed again by the fixation cross for $300 \mathrm{~ms}$. Next the emotional attention and exogenous attention cues were presented together briefly $(50 \mathrm{~ms})$ to avoid any systematic eye movement. Exogenous attention was manipulated by transient thickening of the frame in either the LVF or RVF, which was perceived as a bright flash in the periphery on one side, as done in other standard cognitive paradigms (Berger et al., 2005). Emotional attention was manipulated by presenting an emotional (fearful) face in one frame and a neutral face in the other frame, as done in previous emotional cueing paradigms (Pourtois et al., 2004). Following offset of the face pair and the exogenous visual change, the fixation cross was presented for either 50 or $750 \mathrm{~ms}$, thus introducing a SOA with respect to the cues for emotional and exogenous attention of either 100 (short SOA) or 800 (long SOA) ms. Following this variable time interval, the target, a small rectangle, appeared for $100 \mathrm{~ms}$ in one of the frames, in either the left or the right visual field. Participants were instructed to press " $b$ " on the response keyboard using the index finger of their right hand as fast as possible when they detected the target. Participants had a maximum of $1500 \mathrm{~ms}$ to respond, after which the next trial started. The three attention cueing manipulations were orthogonal. Half of the trials were valid with regards to emotional and exogenous attention, whereas $70 \%$ of the trials were valid with regards to endogenous attention. We also introduced two kinds of catch trials to prevent participants from developing response strategies: in 5\% of the trials no target was presented, and thus no response required; whereas in another $5 \%$ the fixation cross changed to an " $X$ ", instructing participants to press the " $\mathrm{X}$ " key instead of the "b" key.

Data from one female subject had to be excluded from the analysis due to extremely high error rates in the experimental trials $(29.6 \%$, whereas mean error rate for all subjects was 3\%). We analyzed all response times (RTs, computed from target onset) within three standard deviations around the individual mean for correctly detected targets in a repeated-measure ANOVA with the factors emotional attention (valid/invalid) x endogenous attention (valid/invalid) x exogenous attention (valid/invalid) x target hemifield (left/right) x SOA (short/long). Participants generally made very few errors in this task (mean $2.8 \%$ for the experimental trials, mean 10.9 $\%$ for the catch trials), thus we did not analyze the errors. Next, we carried out planned comparisons to further assess the individual validity effects for emotional, endogenous, and exogenous attention, separately for the short and long SOAs. Based on our a priori hypotheses, we also performed two separate four-factorial ANOVAs for trials with short and long SOAs, respectively.

\subsection{Results}

\subsubsection{Overall ANOVA}

As expected, the overall ANOVA revealed faster responses in emotional valid trials $(388 \mathrm{~ms})$ than emotional invalid trials $(391 \mathrm{~ms})$, main effect emotional attention, $F(1$, 
32) $=4.18, p=.013$, partial $\eta^{2}=.19$. This effect was mainly driven by targets presented to the right hemifield (right hemifield, emotional valid: $374 \mathrm{~ms}$, right hemifield, emotional invalid: $380 \mathrm{~ms}$; left hemifield, emotional valid: $402 \mathrm{~ms}$; left hemifield, emotional invalid: $402 \mathrm{~ms}$ ), as indicated by the interaction emotional attention $\mathrm{x}$ target hemifield, $F(1,32)=4.85, p=.035$, partial $\eta^{2}=.13$. The analysis also revealed faster responses in endogenous valid trials $(387 \mathrm{~ms})$ than endogenous invalid trials (392 ms), main effect endogenous attention, $F(1,32)=20.55, p<.001$, partial $\eta^{2}=.39$. Furthermore, responses were faster in exogenous valid trials (388 ms) than in exogenous invalid trials $(391 \mathrm{~ms})$, main effect exogenous attention, $F(1,32)=$ $4.10, p=.051$, partial $\eta^{2}=.11$. Responses were generally faster to targets appearing in the right visual field $(377 \mathrm{~ms})$ than targets appearing in the left visual field (402 ms), as shown by a main effect target hemifield, $F(1,32)=104.04, p<.001$, partial $\eta^{2}=$ .77. Responses were also faster in long SOA trials $(377 \mathrm{~ms})$ than short SOA trials $(402 \mathrm{~ms})$, main effect $S O A, F(1,32)=29.50, p<.001$, partial $\eta^{2}=.48$. This difference was more pronounced for targets appearing in the right visual field than for targets appearing in the left visual field, as indicated by the interaction target hemifield $\mathrm{x} S O A, F(1,32)=15.84, p<.001$, partial $\eta^{2}=.33$. No other two-way or three-way interaction terms reached statistical significance. Planned comparisons (one-tailed t-tests) confirmed a significant validity effect for each of the three attentional manipulations with the short SOA, but only for endogenous attention with the long SOA [Short SOA: emotional attention: $t(32)=2.41, p=.011$, endogenous attention: $t(32)=3.75, p<.001$, exogenous attention: $t(32)=1.94, p=.030$; long SOA: emotional attention: $t(32)<1$, .n.s., endogenous attention: $t(32)=2.08, p=$ .023 , exogenous attention: $t(32)<1$, n.s., all tests one-tailed].

\subsubsection{Short SOA condition}

Confirming the results of the planned comparisons, in the short SOA condition, responses were faster in emotional valid trials $(400 \mathrm{~ms})$ than emotional invalid trials (405 ms), main effect emotional attention, $F(1,32)=5.84, p=.02$, partial $\eta^{2}=.15$; marginally faster in exogenous valid trials (401 ms) than exogenous invalid trials (404 $\mathrm{ms}$ ), main effect exogenous attention, $F(1,32)=3.76, p=.061$, partial $\eta^{2}=.11$; and also faster in endogenous valid trials $(399 \mathrm{~ms}$ ) than endogenous invalid trials (406 $\mathrm{ms}$ ), main effect endogenous attention, $F(1,32)=14.10, p=.001$, partial $\eta^{2}=.31$.

Remarkably, the three attention manipulations were additive, as illustrated in Figure 2 . The decrease in response time as a function of the number of concomitant valid/invalid cues was linear, as revealed by a linear contrast, $F(1,32)=22.49, p$ $<.001$. Furthermore, responses were again faster toward targets presented to the right visual field $(392 \mathrm{~ms})$ than the left visual field (413 ms), main effect target hemifield, $F(1,32)=65.71, p<.001$, partial $\eta^{2}=.67$.

\section{FIGURE 2 ABOUT HERE}

\subsubsection{Long SOA condition}

In the long SOA condition we still found faster RTs in endogenous valid trials (374 $\mathrm{ms})$ than endogenous invalid trials (378 ms), main effect endogenous attention, $F(1$, $32)=4.31, p=.046$, partial $\eta^{2}=.12$. By contrast, neither the emotional attention manipulation (invalid: $377 \mathrm{~ms}$, valid: $376 \mathrm{~ms}, \mathrm{~F}<1$, ns) nor the exogenous attention manipulation (invalid: $377 \mathrm{~ms}$, valid: $376 \mathrm{~ms}, \mathrm{~F}<1$, ns) reached significance. Furthermore, responses were again generally faster toward targets presented to the 
right visual field (361 ms) than toward targets presented to the left visual field (392 $\mathrm{ms})$, main effect target hemifield, $F(1,32)=97.97, p<.001$, partial $\eta^{2}=.75$.

\subsection{Discussion of Experiment 1}

Results of our first experiment showed response facilitations for valid compared to invalid trials for all three attention manipulations, demonstrating that the allocation of attention can be simultaneously influenced by voluntary goals, low-level physical stimulus properties, and emotional stimulus content. The facilitation effects of emotional and exogenous attention were circumscribed to the short SOA (100 ms), consistent with their rapid and reflexive nature, whereas effects of endogenous attention were still present at a longer SOA $(800 \mathrm{~ms})$. In the short SOA condition, the validity effects of different cues added up in a linear fashion (as confirmed by a significant linear effect, see Figure 2), consistent with the assumption of an additive effect of exogenous, endogenous, and emotional attention.

\section{Experiment 2}

In the first study, we investigated the interplay of emotional, endogenous, and exogenous attention, showing that all three attentional subcomponents can operate concurrently. As outlined previously, these different subcomponents may exert their effects via different neural processes, with converging influences on visual perception. Previous neuroimaging work has focused on the relationship of emotional and endogenous attention mechanisms (e.g., Pessoa, 2005; Pourtois et al., 2010; Silvert et al., 2007; Vuilleumier et al., 2001). To investigate the interplay between the two bottom-up reflexive subcomponents emotional and exogenous attention at the neural level, we recorded ERPs to continuously track neural activity during a dot probe task combining manipulations of emotional and exogenous attention. This allowed us to disentangle neural responses related to the different attention manipulations and to different time-windows during a trial. In a typical trial of the dot probe task, the onset of a cue stimulus triggers a rapid shift of attention, which then leads to facilitated processing of a target stimulus if it appears at the validly cued location. The initial rapid attention shift elicited by the cue can be quantified using the N2pc component (Eimer, 1996). The N2pc component reflects shifts of spatial attention related to the selection of lateralized stimuli appearing in the left or right visual field. It is elicited at posterior electrodes between 180 and $300 \mathrm{~ms}$ after stimulus onset in the hemisphere contralateral to the attended stimulus (Holmes, Bradley, Nielsen, \& Mogg, 2009; Luck \& Hillyard, 1994; Woodman \& Luck, 1999). Once attention has been drawn to a location, it increases the perceptual processing of target stimuli appearing at this location. At the neural level, attention enhances the amplitude of the P1 component elicited by an attended stimulus (Luck, Woodman, \& Vogel, 2000). Amplitude modulation of the P1 as a function of the deployment of visuospatial attention is thought to reflect an increase of sensory gain in visual cortex (Hillyard, Vogel, \& Luck, 1998; Pourtois et al., 2004). By jointly analyzing the N2pc component time-locked to cue stimulus onset and the P1 component time-locked to target stimulus onset, we were able to track and disentangle the effects of emotional and exogenous attention during the entire time-course of a dot probe trial - including very rapid effects reflecting the initial attention capture $(\mathrm{N} 2 \mathrm{pc})$, and subsequent effects reflecting the perceptual facilitation (P1) of target processing, appearing at a later point in time. 


\subsection{Methods}

\subsubsection{Participants}

Eighteen University of Geneva students (11 females, mean age 22.3 years)

participated in the EEG experiment. Twelve others ( 8 females, mean age 22.0 years) participated in a behavioral control experiment. All participants were right-handed, had normal or corrected-to-normal vision, and no history of psychiatric or neurological diseases.

\section{FIGURE 3 ABOUT HERE}

\subsubsection{Procedure}

The experiment was similar to Experiment 1, but did not include manipulations of endogenous attention (see Figure 3). The main experiment consisted of one practice block of 8 trials, followed by two experimental blocks with 960 trials in total. A fixation cross was presented for $500 \mathrm{~ms}$. Then, both the emotional and exogenous cue were simultaneously shown for $100 \mathrm{~ms}$. Following offset of the cue, the fixation cross was presented randomly for $100,150,200,250$, or $300 \mathrm{~ms}$. Then the target, a small bar-probe appeared for $150 \mathrm{~ms}$ at the center of one of the two frames, oriented either vertically or horizontally. In emotional valid trials, the target appeared at the location of the emotional face; in exogenous valid trials, the target appeared inside the frame whose size (and thus luminance) had increased. The two attention cueing manipulations were orthogonal, with $50 \%$ of the trials being valid with regards to emotional and exogenous attention, respectively. Participants were asked to judge target orientation as quickly as possible. On each trial, the thickness of one segment within the cross (either the horizontal or vertical line) was slightly increased (from 0.1 to $0.4 \mathrm{~cm}$ ) at the time of the probe onset. Participants were instructed to press the button of the response box using the index finger of their right hand, only when orientation of the target bar matched that of the thicker segment of the fixation cross. The orientation of the thick line at fixation varied from trial to trial and matched that of the bar (50\% horizontal and 50\% vertical) in the periphery in $10 \%$ of the cases. Only matching trials required a button press. We designed a small number of trials that required a motor response $(10 \%)$ to study covert spatial orienting toward emotional stimuli in the vast majority of trials without overt motor response (90\%), therefore minimizing the contamination of motor preparation or execution on EEG signal quality (see Pourtois et al., 2004 for a similar procedure).

We also conducted an additional behavioral control experiment using a target detection task, where participants were instructed to indicate whether the target appeared in the LVF or the RVF, thus increasing the proportion of manual responses from $10 \%$ to $100 \%$ in order to collect enough responses for statistical analyses of the response times.

\subsubsection{EEG Recordings}

EEG was recorded and processed using a Neuroscan 64 channel device (Synamps). Horizontal and vertical EOGs were monitored using four facial bipolar electrodes placed on the outer canthi of the eyes and in the inferior and superior areas of the left orbit. Scalp EEG was recorded from $62 \mathrm{Ag} / \mathrm{AgCl}$ electrodes mounted in a quickcap (extended 10-20 System) with a linked-mastoids reference, amplified with a gain of 
$30 \mathrm{~K}$ and bandpass filtered at $0.01-100 \mathrm{~Hz}$ with a $50 \mathrm{~Hz}$ notch filter. Impedance was kept below $5 \mathrm{k} \Omega$. EEG and EOG were continuously acquired at a rate of $500 \mathrm{~Hz}$ and stored for off-line averaging. EEG was corrected for eye blinks by the subtraction of PCA-transformed EOG components for each electrode, weighted according to VEOG propagation factors. After removal of EEG artifacts (epochs with EEG exceeding +/$75 \mu \mathrm{V}$ were excluded from the averaging), data were segmented around cue onsets (from $-100 \mathrm{~ms}$ to $+700 \mathrm{~ms}$ ) and target onsets (from $-100 \mathrm{~ms}$ to $+500 \mathrm{~ms}$ ). Baseline correction was performed on the 100-ms prestimulus interval. Data were low-pass filtered at $30 \mathrm{~Hz}$. Artifact-free epochs were averaged separately for each electrode, condition, and individual. Bad channels were interpolated using spherical splines. Grand average ERPs were generated by computing mean ERPs across subjects in each condition. Data from two female participants were excluded due to poor quality of electrophysiological recordings.

\subsubsection{Data analysis}

\subsubsection{Behavioral control experiment.}

We analyzed response times (RTs, computed from target onset) within three standard deviations around the individual mean for correctly detected targets in a repeatedmeasure ANOVA with the factors emotional attention (valid/invalid) $\mathrm{x}$ exogenous attention (valid/invalid) x target hemifield (left/right).

\subsubsection{EEG experiment.}

Based on our a priori hypotheses and the previous literature, we analyzed the N2pc component time-locked to cue onset and the P1 component time-locked to target onset, for the valid and invalid conditions of the emotional attention and exogenous attention conditions, respectively. N2pc amplitudes and P1 amplitudes and peak latencies were measured at lateral parietal and parieto-occipital sites, where amplitudes for the components were maximal (P7/P8 for N2pc, PO7/PO8 for P1, see Figures 2 and 3). These sites were selected based on related effects in previous ERP studies (Brosch et al., 2008; Di Russo, Martinez, \& Hillyard, 2003; Holmes et al., 2009; Pourtois et al., 2004) and conspicuous topographic properties of the present ERP data set. Mean amplitudes of the N2pc component (averaged across a time window from $180 \mathrm{~ms}-300 \mathrm{~ms}$ post cue onset) were analyzed using a 2 × 2 × 2 ANOVA with the repeated factors emotional attention (ipsilateral/contralateral), exogenous attention (ipsilateral/contralateral), and electrode position (P7/P8). Peak amplitudes and latencies of the $\mathrm{P} 1$ component (measured during a time window from 80-180 ms post target onset) were analyzed using a $2 \times 2 \times 2 \times 2$ - ANOVA with the repeated factors emotional attention (valid/invalid), exogenous attention (valid/invalid), target hemifield (left/right), and electrode position (PO7/PO8).

\subsection{Results}

\subsubsection{Behavioral performance in the control experiment}

The ANOVA revealed faster responses in emotional valid (340 ms) that invalid (344 ms) trials, main effect emotional attention, $F(1,11)=10.68, p=.007$, partial $\eta^{2}=$ .49; and also faster responses in exogenous valid (334 ms) than invalid (350 ms) trials, main effect exogenous attention, $F(1,11)=11.69, p=.006$, partial $\eta^{2}=.52$. No other main effects or interactions reached statistical significance. 


\subsubsection{N2pc component}

Figure 4A shows ERPs time-locked to cue onset at electrodes P7/P8, located either ipsilateral (black) or contralateral (red) to the location of the exogenous cue (i.e., the black line reflects the average of electrode P7 [left hemisphere] when the exogenous cue was presented to the LVF and of electrode P8 [right hemisphere] when the cue was presented to the RVF). Figure 2B shows ERPs time-locked to cue onset at electrodes P7/P8 ipsilateral (black) and contralateral (red) to the emotional cue locations.

As can be seen in Figure 4A, an enhanced negativity appeared on the scalp contralateral to the exogenous cues, reflecting a shift of attention toward the exogenous cue. ANOVA confirmed that the mean amplitude of the N2pc (180 ms $300 \mathrm{~ms}$ ) was significantly more negative at electrodes contralateral to the exogenous cue $(-2.27 \mu \mathrm{V})$ than at electrodes ipsilateral to the exogenous cue $(-1.87 \mu \mathrm{V})$, main effect exogenous attention, $F(1,15)=7.42, p=.017$, partial $\eta^{2}=.33$. No such difference was observed as a function of the emotional cues (contralateral electrodes: $-2.12 \mu \mathrm{V}$, ipsilateral electrodes: $-2.01 \mu \mathrm{V}, F(1,15)=0.54, \mathrm{~ns})$. No other main effects or interactions reached statistical significance.

\section{FIGURE 4 ABOUT HERE}

\subsubsection{P1 component}

Figure 5A shows ERPs time-locked to target stimulus onset in exogenous invalid (black) and exogenous valid (red) trials, Figure 5B shows ERPs time-locked to target stimulus onset in emotional invalid (black) and emotional valid (red) trials at electrode PO8. ANOVA revealed that the amplitude of the P1 component was larger for targets that had been validly cued by the emotional stimulus $(3.35 \mu \mathrm{V})$ than for targets invalidly cued by the emotional stimulus $(3.09 \mu \mathrm{V})$, main effect emotional attention, $F(1,15)=6.12, p=.025$, partial $\eta^{2}=.29$, reflecting an increase of sensory gain in visual cortex for targets following an emotional cue stimulus. No such difference was observed as a function of the exogenous cues (valid trials: $3.2 \mu \mathrm{V}$, invalid trials: $3.2 \mu \mathrm{V}, F(1,15)=0.25$, ns $)$.

Furthermore, P1 amplitudes were larger to targets presented to the left hemifield (3.5 $\mu \mathrm{V})$ than to the right hemifield $(2.9 \mu \mathrm{V})$, main effect target hemifield, $F(1,15)=$ $13.48, p=.002$ partial $\eta^{2}=.47$, mainly driven by the right cerebral hemisphere (PO7 target left: $2.95 \mu \mathrm{V}$, PO7 target right: $2.83 \mu \mathrm{V}$, PO8 target left: $4.11 \mu \mathrm{V}$, PO8 target right: $3.0 \mu \mathrm{V}$, interaction target hemifield $\mathrm{x}$ electrode position, $F[1,15]=22.90, p<$ .001 , partial $\eta^{2}=.60$ ).

With regards to the latencies of the peak of the P1 component, an interaction of target hemifield $\mathrm{x}$ electrode position, $F(1,15)=6.0, p=.027$, partial $\eta^{2}=.29$, reflected that the P1 peaked earlier at electrodes contralateral to the target hemifield (PO7 target left hemifield: $130 \mathrm{~ms}$, PO7 target right hemifield: $124 \mathrm{~ms}$, PO8 target left hemifield: 128 ms, PO8 target right hemifield: $140 \mathrm{~ms}$ ). No other main effects or interactions reached statistical significance. 


\subsection{Discussion of Experiment 2}

The response time data revealed response facilitation in valid compared to invalid trials with respect to both the exogenous attention manipulation and the emotional attention manipulation, replicating our finding from Experiment 1. This indicates that both the low-level physical properties of a stimulus and its emotional value influence the allocation of attention, even when these cues are not predictive of target side (50\% validity). However, the electrophysiological data revealed a double dissociation with regards to the ERP components and thus the temporal loci at which the two attention manipulations presumably exerted their effects. The exogenous attention cue led to an enhanced $\mathrm{N} 2 \mathrm{pc}$ component at contralateral electrodes, reflecting a rapid initial attentional capture by the luminance change, whereas the emotional attention cue did not lead to a significant enhancement at the level of the N2pc component. The opposite pattern was observed for the target-locked P1 component. Targets that had been validly cued by an emotional stimulus elicited a larger P1 than invalidly cued targets, reflecting an increase of sensory gain in visual cortex for stimuli appearing at the previous location of an emotional stimulus. In contrast, the exogenous luminance cue did not lead to a significant enhancement of the P1 component for the subsequent target. Taken together, these results suggest that, while both low-level physical properties of a stimulus and its emotional value influence the rapid allocation of attention, two separate mechanisms may be operating in parallel to increase neural processing of these different types of behaviorally relevant stimuli. Effects of exogenous attention may begin rapidly and primarily influence early stages of processing (fast attention orienting effect, see, e.g., Brignani, Guzzon, Marzi, \& Miniussi, 2009), but be more short-lived; whereas the effects of emotional attention may be expressed at a slightly later onset and mainly impact on the visual encoding of the subsequent target as a function of the preceding emotional information at the same location (gain control effect, see Hillyard et al., 1998).

\section{General Discussion}

We report results from two studies investigating the interplay of the attentional subcomponents related to emotional, endogenous and exogenous orienting by orthogonally manipulating them in a dot probe paradigm. In both experiments, we observed faster behavioral responses for target detection in valid than invalid conditions for each type of attentional manipulation, suggesting that multiple separate attention mechanisms can operate simultaneously on visual perception.

In addition, the neurophysiological data from Experiment 2 revealed a dissociation of the neural processes underlying the effects of exogenous and emotional attention. Selective effects of exogenous attention were present at the level of the cue-locked $\mathrm{N} 2 \mathrm{pc}$ component, which reflects attentional capture by a stimulus (here the abrupt change in luminance). In contrast, the emotional value of the cue mainly exerted an effect at the level of the target-locked P1 component, with targets following an emotional cue eliciting larger P1 amplitudes than targets following a neutral stimulus, thus reflecting an increase of sensory gain in visual cortex for emotionally cued stimuli. This gain increase may facilitate perceptual processing of subsequently appearing stimuli, as demonstrated for example by increased contrast sensitivity following the presentation of fearful faces (Phelps et al., 2006). The 
electrophysiological dissociation observed here suggests that, while both low-level physical properties of a stimulus and its emotional value influence the allocation of attentional resources, two separate neural mechanisms may be active to bias perceptual processing toward different aspects of encountered stimuli.

The observed difference in the time course of the unfolding of these attention subprocesses may potentially be related to the multiplicity and versatility of selection mechanisms operating on conscious visual perception. Exogenous attention allocation can be triggered by low-level physical stimulus intensity, which is directly represented by the initial strength of the neural activation, and therefore does not need additional processing steps before attentional prioritization can take place. The emotional relevance of a stimulus may be based on evolutionary prepared, strongly conditioned or highly overlearned stimulus classes from the personal learning history (Brosch, 2009). In any case, affective evaluation of incoming stimuli results from the reactivation of stored representations, either via explicit knowledge or via implicit pathways (Brosch, Pourtois, \& Sander, 2010). At the neural systems level, the effects of emotional attention in task such as the dot probe may be implemented by the amygdala, which, once it has detected the potential emotional relevance of a stimulus (Sander, Grafman, \& Zalla, 2003) will send biasing signals to early cortical visual and/or fronto-parietal regions responsible for orienting and shifting attention in space, so that subsequent information arising at the same location as emotional cues will also benefit from enhanced processing resources (Pourtois, Schwartz, Seghier, Lazeyras, \& Vuilleumier, 2006). Thus, as the computation of emotional attention involves an additional processing step, emotional attention mechanisms may need more time than exogenous attention effects to exert an impact on extrastriate visual cortex activations (which are likely underlying the occipital ERP components recorded in this study).

The neural dissociation observed in our study may also reflect different modulatory effects that attention mechanisms could exert in paradigms such as the dot probe task. These include attention capture, describing the rapid direction of attention toward a stimulus, and attention disengagement, referring to the prolonged dwelling time on a stimulus before attention can "move on" to explore other stimuli (Weierich, Treat, \& Hollingworth, 2008). Some authors have claimed that the emotional attention effects in the dot probe task are primarily related to the disengagement component (Fox et al., 2002). In this task, rapid exogenous capture may be reflected at the level of the cue $\mathrm{N} 2 \mathrm{pc}$, whereas an increased dwelling time due to emotional attention may be reflected at the level of the later target P1. Note also that RT difference between conditions are unlikely to reflect a general slowing in the presence of emotional stimuli as this effect has been observed when face cues are presented unilaterally (see Mogg, Holmes, Garner, \& Bradley, 2008), unlike our paradigm where fearful faces are always accompanied simultaneously with a neutral cue on the other side.

The selectivity of the exogenous and emotional attention modulation with regards to the ERP components seems however to be specific to the current task which involves a concurrent manipulation of exogenous and emotional attention. Some studies manipulating only one of the attention subcomponent have observed effects of emotional attention at the level of the cue-locked N2pc (see Holmes et al., 2009; note however that in their study the emotional cue was presented for $500 \mathrm{~ms}$, which is five times longer than the presentation time of the current study). Other studies found effects of exogenous attention at the level of the target-locked P1 (e.g., Heinze, Luck, 
Mangun, \& Hillyard, 1990). Thus, there may be some form of interaction or competition between the different attention subcomponents when manipulated concurrently, in that the effects of the different subcomponents become more temporally constrained, or differentially apparent in the EEG recording.

Previous work supports the assumption that distinct attentional subcomponents can operate independently under some circumstances but interfere under others, depending on specific processing stages, task demands and sensory modalities. For example, exogenous and endogenous attention can interact under certain conditions (Berger et al., 2005; Yantis \& Jonides, 1990), and ERP studies have demonstrated both independent and interacting effects of exogenous and endogenous attention, arising at different time points (Hopfinger \& West, 2006). Furthermore, neural responses to emotional stimuli are prolonged (e.g. $>500 \mathrm{~ms}$ post-onset) when endogenous attentional resources are available, but briefer (e.g. $<200 \mathrm{~ms}$ ) when endogenous attention is diverted by another concurrent task (Eimer \& Holmes, 2007). Likewise, although emotional stimuli may evoke reflexive and involuntary processing under many conditions, this does not preclude that such effects can be amplified or attenuated by factors such as anxiety or attentional control capacity (Bishop et al., 2007). In addition, experiments using crossmodal manipulations have demonstrated interactions between emotional and exogenous attention in the form of decreased P3 amplitude to auditory startle probes when emotionally intense visual stimuli are presented concurrently (Cuthbert, Schupp, Bradley, McManis, \& Lang, 1998; Keil et al., 2007). In animal work, crossmodal interactions between different attention systems may even affect sensory processing at the level of sensory transduction (e.g., in the cochlea, see Delano, Elgueda, Hamame, \& Robles, 2007). Altogether, these findings suggest that the different attention systems may initially operate relatively independently (i.e. not tap into each other's resources), but may compete and interact at later time points (e.g., at the level of the P3 component) or when different sensory modalities are integrated. Ultimately, all attention subcomponents serve the optimization of environmental information processing by selecting potentially relevant information for further cognitive processing. The demonstration that subcomponents can operate partially independently does not undermine the need to eventually integrate their outputs, as their combined influences may converge on common perceptual and cognitive systems, allowing a selective enhancement of the neural representation of relevant information among competing inputs.

Taken together, our findings suggest that parallel processes operate to increase the neural processing of information concerning voluntary goals and expectations, emotionally relevant events, and sudden changes in the physical stimulus properties, respectively. Efficient systems for the rapid detection of various features of potentially important stimuli provide adaptive mechanisms for responding to behaviorally relevant information in a multifarious environment. Our findings support the hypothesis that emotional attention is mediated by neural systems being partly independent of exogenous and endogenous attention (e.g., Lucas \& Vuilleumier, 2008). It has been proposed that emotional attention involves a specialized brain circuitry centered around the amygdala (Vuilleumier, 2005), and thus should be at least partly separable from endogenous and exogenous attention, which both primarily implicate fronto-parietal networks of cortical regions (Corbetta \& Shulman, 2002). Our findings are thus consistent with this neuro-cognitive model. 
Further research is needed to better delineate under which circumstances the attentional subcomponents do operate independently or do interact, as well as to identify which neural pathways and which temporal sequences are involved. Specific task requirements, goals, or internal states might influence the extent to which the systems operate independently. In order to further explore the concurrent processing of the different types of environmental and internal information, different tasks varying cognitive and effective demands as well as stimulus characteristics should be employed in both healthy participants and specific patient populations, and combination of EEG and fMRI should be used to establish the corresponding neural correlates of these effects.

\section{Acknowledgements}

This work was supported by the National Centre of Competence in Research (NCCR) Affective Sciences, financed by the Swiss National Science Foundation (SNSF, $\mathrm{n}^{\circ}$ 51NF40-104897) and hosted by the University of Geneva, and by a SNSF fellowship for advanced researchers to T.B. ( ${ }^{\circ}$ PA00P1_131435). We want to thank Susanne Phillip for helping with the behavioral control study.

\section{References}

Amaral, D. G., Behniea, H., \& Kelly, J. L. (2003). Topographic organization of projections from the amygdala to the visual cortex in the macaque monkey. Neuroscience, 118, 1099-1120.

Berger, A., Henik, A., \& Rafal, R. (2005). Competition between endogenous and exogenous orienting of visual attention. Journal of Experimental Psychology: General, 134, 207-221.

Bishop, S. J. (2007). Neurocognitive mechanisms of anxiety: an integrative account. Trends in Cognitive Sciences, 11, 307-316.

Bishop, S. J., Duncan, J., Brett, M., \& Lawrence, A. D. (2004). Prefrontal cortical function and anxiety: controlling attention to threat-related stimuli. Nature Neuroscience, 7, 184-188.

Bishop, S. J., Jenkins, R., \& Lawrence, A. D. (2007). Neural processing of fearful faces: effects of anxiety are gated by perceptual capacity limitations. Cerebral Cortex, 17, 1595-1603.

Bradley, B. P., Mogg, K., \& Millar, N. H. (2000). Covert and overt orienting of attention to emotional faces in anxiety. Cognition and Emotion, 14, 789-808.

Brignani, D., Guzzon, D., Marzi, C. A., \& Miniussi, C. (2009). Attentional orienting induced by arrows and eye-gaze compared with an endogenous cue. Neuropsychologia, 47, 370-381.

Brosch, T. (2009). Intrinsic pleasantness. In D. Sander \& K. R. Scherer (Eds.), The Oxford Companion to Emotion and the Affective Sciences (pp. 225-226). Oxford: Oxford University Press.

Brosch, T., Pourtois, G., \& Sander, D. (2010). The perception and categorization of emotional stimuli: A review. Cognition and Emotion, 24, 377-400.

Brosch, T., Sander, D., Pourtois, G., \& Scherer, K. R. (2008). Beyond fear: Rapid spatial orienting towards positive emotional stimuli. Psychological Science, $19,362-370$.

Brosch, T., Sander, D., \& Scherer, K. R. (2007). That baby caught my eye... Attention capture by infant faces. Emotion, 7, 685-689. 
Compton, R. J. (2003). The interface between emotion and attention: A review of evidence from psychology and neuroscience. Behavioral and Cognitive Neuroscience Reviews, 2, 115-129.

Corbetta, M., Patel, G., \& Shulman, G. L. (2008). The reorienting system of the human brain: from environment to theory of mind. Neuron, 58, 306-324.

Corbetta, M., \& Shulman, G. L. (2002). Control of goal-directed and stimulus-driven attention in the brain. Nature Reviews Neuroscience, 3, 201-215.

Cuthbert, B. N., Schupp, H. T., Bradley, M., McManis, M., \& Lang, P. J. (1998). Probing affective pictures: attended startle and tone probes. Psychophysiology, 35, 344-347.

Delano, P. H., Elgueda, D., Hamame, C. M., \& Robles, L. (2007). Selective attention to visual stimuli reduces cochlear sensitivity in chinchillas. Journal of Neuroscience, 27, 4146-4153.

Desimone, R., \& Duncan, J. (1995). Neural mechanisms of selective visual attention. Annual Review of Neuroscience, 18, 193-222.

Di Russo, F., Martinez, A., \& Hillyard, S. A. (2003). Source analysis of event-related cortical activity during visuo-spatial attention. Cerebral Cortex, 13, 486-499.

Driver, J. (2001). A selective review of selective attention research from the past century. British Journal of Psychology, 92, 53-78.

Duncan, J. (1980). The demonstration of capacity limitation. Cognitive Psychology, 12.

Egeth, H. E., \& Yantis, S. (1997). Visual attention: control, representation, and time course. Annual Review of Psychology, 48, 269-297.

Eimer, M. (1996). The N2pc component as an indicator of attentional selectivity. Electroencephalography and Clinical Neurophysiology, 99, 225-234.

Eimer, M., \& Holmes, A. (2007). Event-related brain potential correlates of emotional face processing. Neuropsychologia, 45, 15-31.

Fox, E., Russo, R., Bowles, R., \& Dutton, K. (2001). Do threatening stimuli draw or hold visual attention in subclinical anxiety? Journal of Experimental Psychology: General, 130, 681-700.

Fox, E., Russo, R., \& Dutton, K. (2002). Attentional bias for threat: Evidence for delayed disengagement from emotional faces. Cognition and Emotion, 16, 355-379.

Grandjean, D., Sander, D., Pourtois, G., Schwartz, S., Seghier, M. L., Scherer, K. R., et al. (2005). The voices of wrath: Brain responses to angry prosody in meaningless speech. Nature Neuroscience, 8, 145-146.

Heinze, H. J., Luck, S. J., Mangun, G. R., \& Hillyard, S. A. (1990). Visual eventrelated potentials index focused attention within bilateral stimulus arrays. I. Evidence for early selection. Electroencephalography and Clinical Neurophysiology, 75, 511-527.

Hillyard, S. A., Vogel, E. K., \& Luck, S. J. (1998). Sensory gain control (amplification) as a mechanism of selective attention: Electrophysiological and neuroimaging evidence. Philosophical Transactions of the Royal Society B: Biological Sciences, 353, 1257-1270.

Holmes, A., Bradley, B. P., Nielsen, M. K., \& Mogg, K. (2009). Attentional selectivity for emotional faces: Evidence from human electrophysiology. Psychophysiology, 46, 62-68.

Hopfinger, J. B., \& West, V. M. (2006). Interactions between endogenous and exogenous attention on cortical visual processing. Neuroimage, 31, 774-789. 
Jonides, J. (1981). Voluntary versus automatic control over the mind's eye's movement. In J. B. Long \& A. Baddeley (Eds.), Attention and Performance (Vol. IX, pp. 187-203). Hillsdale, NJ: Erlbaum.

Keil, A., Bradley, M. M., Junghofer, M., Russmann, T., Lowenthal, W., \& Lang, P. J. (2007). Cross-modal attention capture by affective stimuli: evidence from event-related potentials. Cognitive Affective and Behavioral Neuroscience, 7, $18-24$.

Keil, A., Moratti, S., Sabatinelli, D., Bradley, M. M., \& Lang, P. J. (2005). Additive effects of emotional content and spatial selective attention on electrocortical facilitation. Cerebral Cortex, 15, 1187-1197.

Lang, P. J., Bradley, M. M., \& Cuthbert, B. N. (1997). Motivated attention: Affect, activation, and action. In P. J. Lang, R. F. Simons \& M. T. Balaban (Eds.), Attention and orienting: Sensory and motivational processes (pp. 97-135). Mahwah, NJ: Lawrence Erlbaum Associates Publishers.

LeDoux, J. E. (2000). Emotion circuits in the brain. Annual Review of Neuroscience, $23,155-184$.

Lucas, N., \& Vuilleumier, P. (2008). Effects of emotional and non-emotional cues on visual search in neglect patients: Evidence for distinct sources of attentional guidance. Neuropsychologia, 46, 1401-1414.

Luck, S. J., \& Hillyard, S. A. (1994). Spatial filtering during visual search: Evidence from human electrophysiology. Journal of Experimental Psychology: Human Perception and Performance, 20, 1000-1014.

Luck, S. J., Woodman, G. F., \& Vogel, E. K. (2000). Event-related potential studies of attention. Trends in Cognitive Sciences, 4, 432-440.

MacLeod, C., Mathews, A., \& Tata, P. (1986). Attentional bias in emotional disorders. Journal of Abnormal Psychology, 95, 15-20.

Marois, R., \& Ivanoff, J. (2005). Capacity limits of information processing in the brain. Trends in Cognitive Sciences, 9, 296-305.

Mogg, K., \& Bradley, B. P. (1999). Orienting of attention to threatening facial expressions presented under conditions of restricted awareness. Cognition and Emotion, 13, 713-740.

Mogg, K., Holmes, A., Garner, M., \& Bradley, B. P. (2008). Effects of threat cues on attentional shifting, disengagement and response slowing in anxious individuals. Behaviour Research and Therapy, 46, 656-667.

Öhman, A., Flykt, A., \& Esteves, F. (2001). Emotion drives attention: Detecting the snake in the grass. Journal of Experimental Psychology: General, 130, 466478.

Peelen, M., Heslenfeld, D. J., \& Theeuwes, J. (2004). Endogenous and exogenous attention shifts are mediated by the same large-scale neural network. Neuroimage, 22, 822-830.

Pessoa, L. (2005). To what extent are emotional visual stimuli processed without attention and awareness? Current Opinion in Neurobiology, 15, 188-196.

Pessoa, L., McKenna, M., Gutierrez, E., \& Ungerleider, L. G. (2002). Neural processing of emotional faces requires attention. Proceedings of the National Academy of Sciences of the United States of America, 99, 11458-11463.

Pessoa, L., Padmala, S., \& Morland, T. (2005). Fate of unattended fearful faces in the amygdala is determined by both attentional resources and cognitive modulation. Neuroimage, 28, 249-255. 
Phelps, E. A., Ling, S., \& Carrasco, M. (2006). Emotion facilitates perception and potentiates the perceptual benefits of attention. Psychological Science, 17, 292-299.

Posner, M. I., Snyder, C. R., \& Davidson, B. J. (1980). Attention and the detection of signals. Journal of Experimental Psychology, 109, 160-174.

Pourtois, G., Grandjean, D., Sander, D., \& Vuilleumier, P. (2004).

Electrophysiological correlates of rapid spatial orienting towards fearful faces. Cerebral Cortex, 14, 619-633.

Pourtois, G., Schwartz, S., Seghier, M. L., Lazeyras, F., \& Vuilleumier, P. (2006). Neural systems for orienting attention to the location of threat signals: an event-related fMRI study. Neuroimage, 31, 920-933.

Pourtois, G., Spinelli, L., Seeck, M., \& Vuilleumier, P. (2010). Temporal precedence of emotion over attention modulations in the lateral amygdala: Intracranial ERP evidence from a patient with temporal lobe epilepsy. Cognitive, Affective, \& Behavioral Neuroscience, 10, 83-93.

Pourtois, G., Thut, G., Grave de Peralta, R., Michel, C., \& Vuilleumier, P. (2005). Two electrophysiological stages of spatial orienting towards fearful faces: Early temporo-parietal activation preceding gain control in extrastriate visual cortex. Neuroimage, 26, 149-163.

Sander, D., Grafman, J., \& Zalla, T. (2003). The human amygdala: An evolved system for relevance detection. Reviews in the Neurosciences, 14, 303-316.

Sander, D., Grandjean, D., Pourtois, G., Schwartz, S., Seghier, M. L., Scherer, K. R., et al. (2005). Emotion and attention interactions in social cognition: brain regions involved in processing anger prosody. Neuroimage, 28, 848-858.

Shepherd, M., \& Muller, H. J. (1989). Movement versus focusing of visual attention. Perception \& Psychophysics, 46, 146-154.

Silvert, L., Lepsien, J., Fragopanagos, N., Goolsby, B., Kiss, M., Taylor, J. G., et al. (2007). Influence of attentional demands on the processing of emotional facial expressions in the amygdala. Neuroimage, 38, 357-366.

Theeuwes, J. (1991). Exogenous and endogenous control of attention: the effect of visual onsets and offsets. Perception and Psychphysics, 49, 83-90.

Tipples, J., \& Sharma, D. (2000). Orienting to exogenous cues and attentional bias to affective pictures reflect separate processes. British Journal of Psychology, 91, 87-97.

Vuilleumier, P. (2005). How brains beware: Neural mechanisms of emotional attention. Trends in Cognitive Sciences, 9, 585-594.

Vuilleumier, P., Armony, J. L., Driver, J., \& Dolan, R. J. (2001). Effects of attention and emotion on face processing in the human brain: An event-related fMRI study. Neuron, 30, 829-841.

Vuilleumier, P., \& Brosch, T. (2009). Interactions of emotion and attention. In M. S. Gazzaniga (Ed.), The Cognitive Neurosciences IV (pp. 925-934). Cambridge: MIT Press.

Vuilleumier, P., \& Driver, J. (2007). Modulation of visual processing by attention and emotion: Windows on causal interactions between human brain regions. Philosophical Transactions of the Royal Society B: Biological Sciences, 362, 837-855.

Vuilleumier, P., Richardson, M. P., Armony, J. L., Driver, J., \& Dolan, R. J. (2004). Distant influences of amygdala lesion on visual cortical activation during emotional face processing. Nature Neuroscience, 7, 1271-1278. 
Weierich, M. R., Treat, T. A., \& Hollingworth, A. (2008). Theories and measurement of visual attentional processing in anxiety. Cognition and Emotion, 22, 9851018.

Williams, L. M., \& Gordon, E. (2007). Dynamic organization of the emotional brain: responsivity, stability, and instability. Neuroscientist, 13, 349-370.

Wolfe, J. M., \& Horowitz, T. S. (2004). What attributes guide the deployment of visual attention and how do they do it? Nature Reviews Neuroscience, 5, 495501.

Woodman, G. F., \& Luck, S. J. (1999). Electrophysiological measurement of rapid shifts of attention during visual search. Nature, 400, 867-869.

Yantis, S., \& Jonides, J. (1990). Abrupt visual onsets and selective attention: Voluntary versus automatic allocation. Journal of Experimental Psychology: Human Perception and Performance, 16, 121-134. 


\section{Figure captions}

Figure 1. Experimental sequence of Experiment 1.

Figure 2. Linear additive effects of emotional, exogenous, and endogenous attention manipulations. Response times are plotted in milliseconds (with S.E.) for targets that were (a) invalidly cued by all three attention manipulations, (b) invalidly cued by two attention manipulations (but validly cued by one manipulation, either exogenous, endogenous, or emotional), (c) invalidly cued by one attention manipulation and validly cued by two attention manipulations, and (d) validly cued by all three attention manipulations in trials with short SOA. Response times decreased with increasing valid cues. The decrease in response time as a function of the number of concomitant valid cues was linear, as revealed by a linear contrast, $F(1,32)=22.49, p<.001$.

Figure 3. Experimental sequence of Experiment 2.

Figure 4. N2pc component time-locked to the onset of the cue stimulus. (A) Enhanced $\mathrm{N} 2 \mathrm{pc}$ component at electrodes contralateral to the exogenous attention cue reflecting a rapid initial attention shift toward the exogenous cue. (B) In contrast, no modulation of the N2pc component by the emotional attention cue was observed.

Figure 5. P1 component time-locked to the onset of the target stimulus. (A) No modulation of target processing by the exogenous cue was observed at the level of the P1 component. (B) Enhanced P1 component elicited by targets that have been validly cued by emotional stimuli reflecting an increase of sensory gain in visual cortex. 


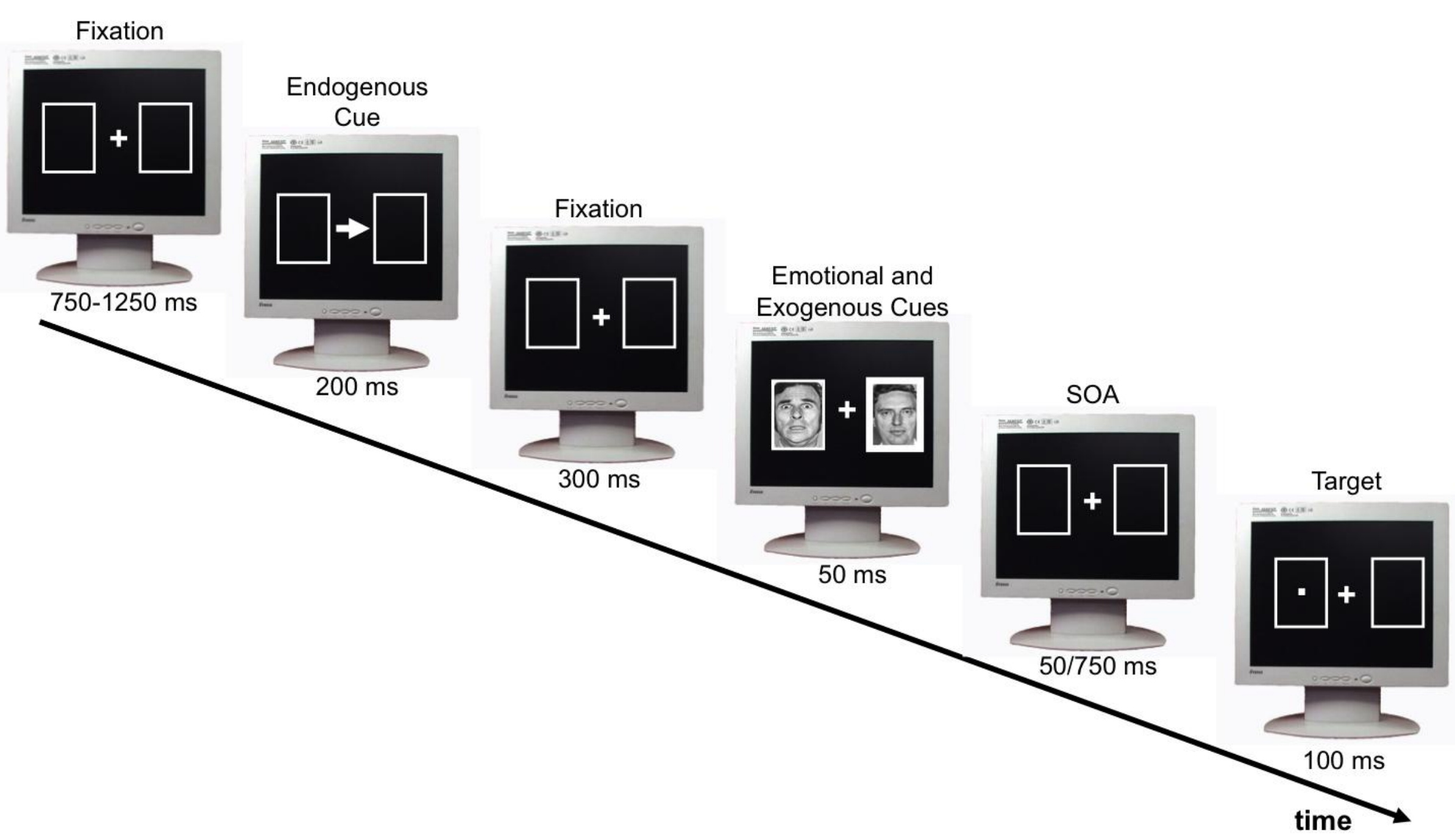

Figure 1 


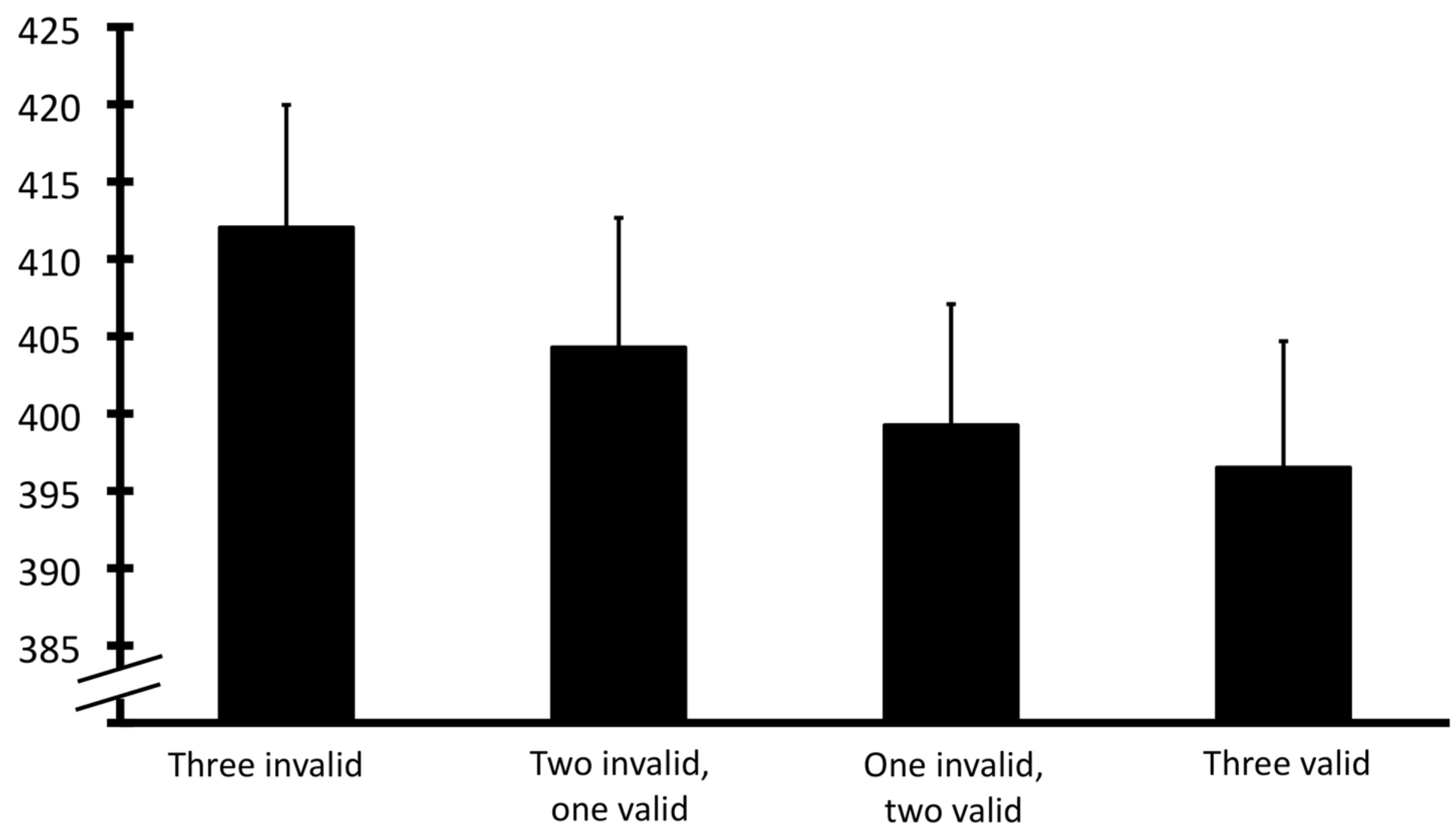

Figure 2 
Fixation

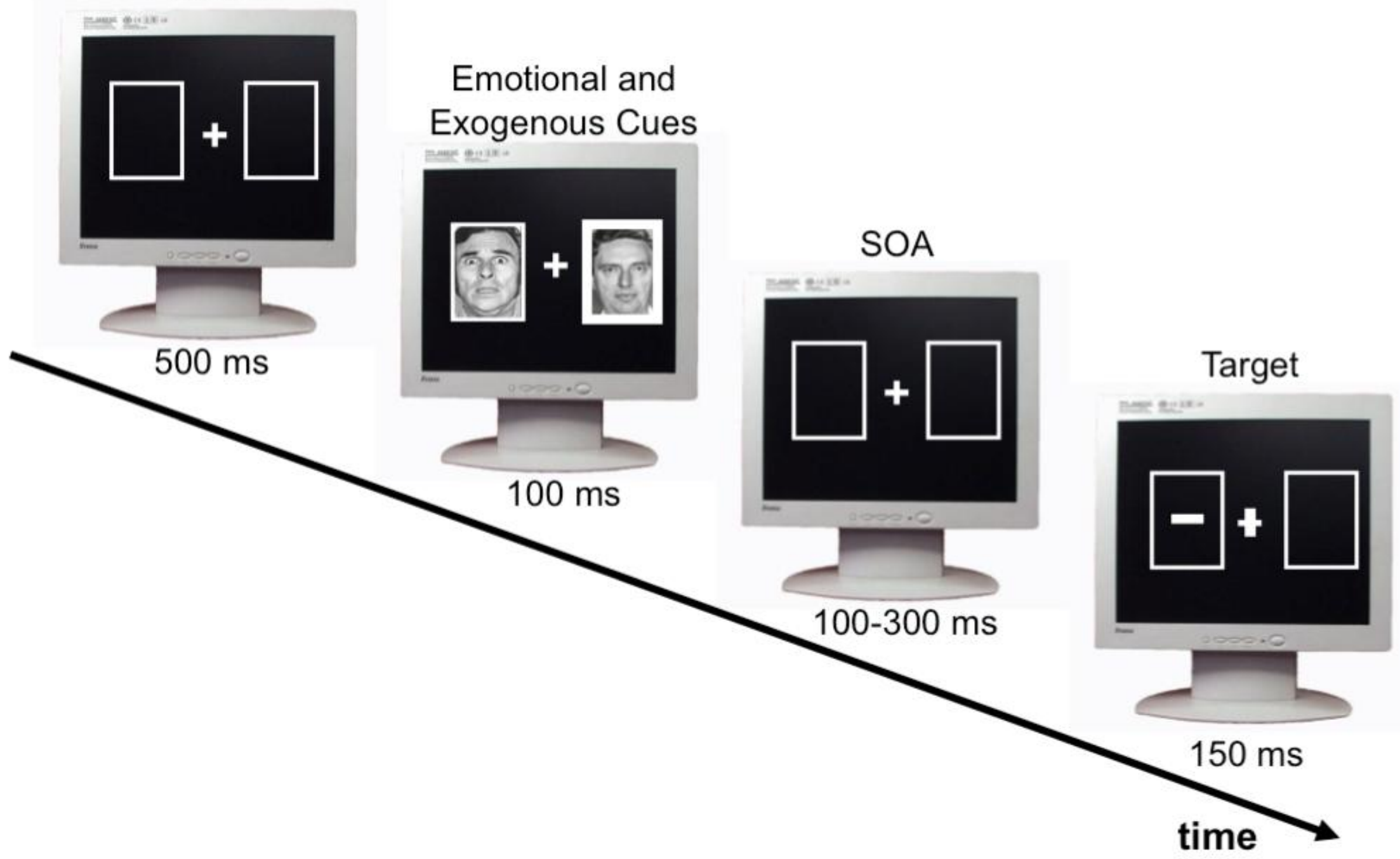

Figure 3 
A

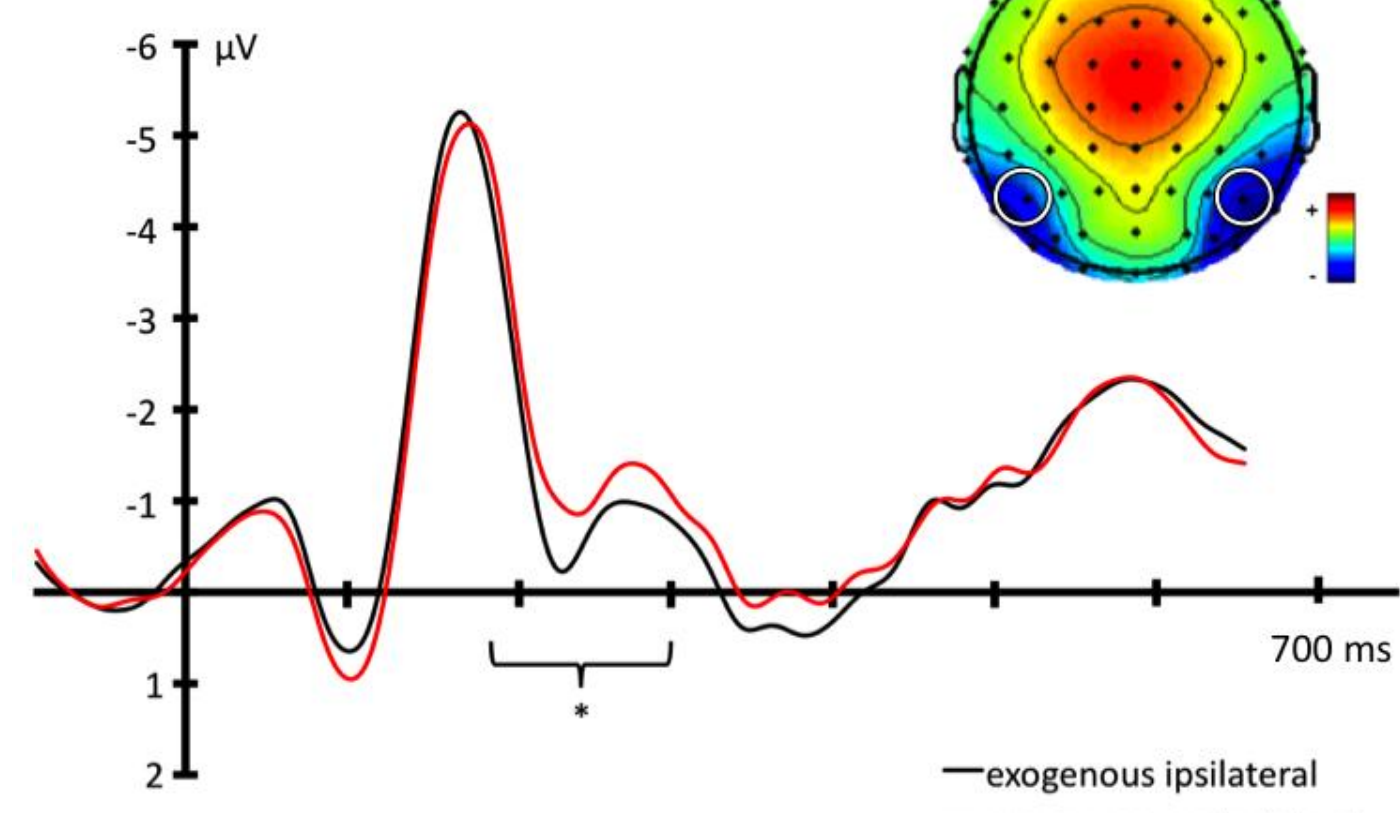

B

- exogenous contralateral

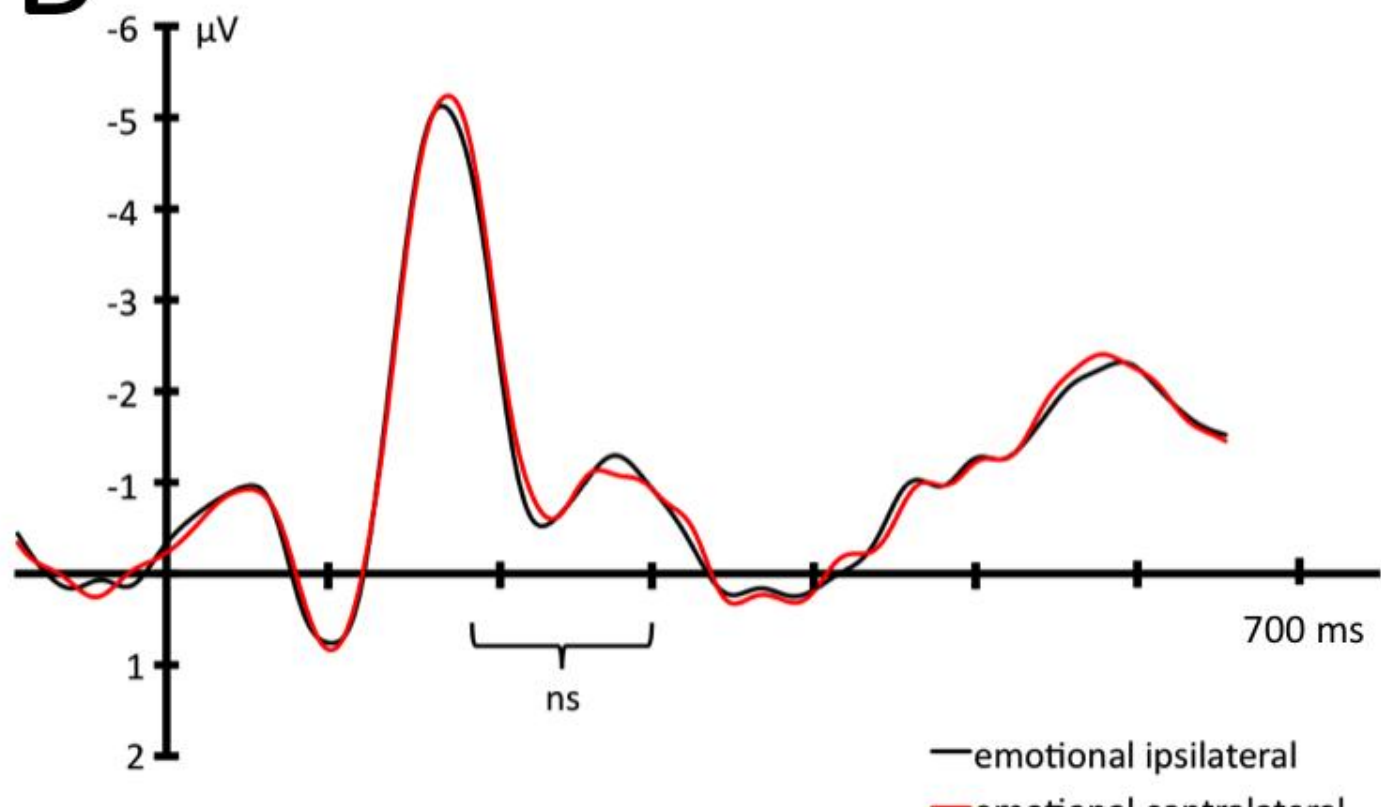

—emotional contralateral

Figure 4 


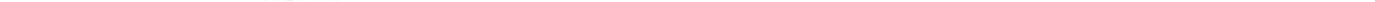

Figure 5 